\title{
Extase et hystérie : le ravissement musical dans les romans de Dumas
}

Julie Anselmini

\section{OpenEdition}

12 Journals

Édition électronique

URL : http://journals.openedition.org/recherchestravaux/448

DOI : 10.4000/recherchestravaux.448

ISSN : 1969-6434

Éditeur

UGA Éditions/Université Grenoble Alpes

Édition imprimée

Date de publication : 15 mai 2011

Pagination : 69-80

ISBN : 978-2-84310-200-4

ISSN : 0151-1874

\section{Référence électronique}

Julie Anselmini, «Extase et hystérie : le ravissement musical dans les romans de Dumas », Recherches \& Travaux [En ligne], 78 | 2011, mis en ligne le 15 novembre 2012, consulté le 08 septembre 2020. URL : http://journals.openedition.org/recherchestravaux/448 ; DOI : https://doi.org/10.4000/ recherchestravaux.448 
Julie Anselmini

Université de Caen

\section{Extase et hystérie : le ravissement musical dans les romans de Dumas}

Choisir l'exemple de Dumas (père) pour illustrer la haine de la musique, ou les vices qui lui sont imputés, pourrait sembler paradoxal. D'une part, en effet, cet auteur est bien loin de détester la musique. Grand amateur de Mozart ou de Weber, qu'il cite souvent dans ses romans, il a lui-même utilisé la musique dans sa production scénique, en écrivant des opéras comme Piquillo (en collaboration avec Nerval), créé à l'Opéra-Comique en octobre I837' ; il a par ailleurs eu pour maîtresse la cantatrice Caroline Ungher, véritable diva de l'opéra romantique ${ }^{2}$, et il a fréquenté de nombreux musiciens : Liszt, rencontré aux réunions de l'Arsenal, chez Charles Nodier, mais aussi Berlioz, dont il fut un grand $\mathrm{ami}^{3}$, ou encore Rossini, avec qui il n'hésite pas à se comparer, évoquant

[...] ce nom mélodieux qui représente autant de millions de rondes, de blanches, de noires, de croches, de doubles croches, de triples croches, de bémols, de dièses, de bécarres, que [s]on nom à [lui] représente de millions de lettres ${ }^{4}$.

I. Dumas reviendra au théâtre musical avec deux autres «opéras-comiques", Thaïs, en 1858 , et Le Roman d'Elvire, en I86o. On ne lui connaît qu'une tentative dans le genre "sérieux", Samson, en 1856 .

2. Beethoven la choisit pour créer la Neuvième Symphonie et la Missa solemnis. Elle chanta sur toutes les scènes d'Europe : Norma de Bellini, Otello de Rossini, Don Giovanni de Mozart, avant de créer à Venise le Belisario de Donizetti. Dumas la rencontra à Naples en I835.

3. Voir R. L. Evans, Les Romantiques français et la musique, Honoré Champion, I934; rééd. Slatkine Reprints, I976, p. IIO et II4.

4. Lettres sur la cuisine à un prétendu gourmand napolitain, Mercure de France, 1996, p. 4I. 
D'autre part, de façon plus large, le XIx e siècle apparaît comme l'âge d'or de la musique occidentale : "art privilégié de ce siècles", selon Pierre de Lahalle, la musique prend alors valeur de "modèle référentiel, culturel, artistique ${ }^{6}$; elle est centrale dans l'épistémè romantique.

Force est pourtant de constater qu'à plusieurs reprises, dans les romans de Dumas, la musique est à l'origine de scènes paroxystiques où un personnage - toujours une jeune fille - se voit dépossédé de lui-même et jeté dans un monde inconnu, pour son plus grand malheur. Dans Pauline (I838), l'héroïne éponyme s'évanouit ainsi après avoir chanté le duo du premier acte du Don Giovanni de Mozart avec le comte Horace de Beuzeval, qui la poursuit de ses assiduités. Elle racontera plus tard :

Je ne puis vous dire ce qu'il y avait d'amour dans la voix du comte, lorsqu'il reprit : vieni, mio bel diletto, et ce qu'il mit de séduction et de promesses dans cette phrase : io cangerò tua sorte; tout cela était si applicable à moi, ce duo semblait si bien choisi pour la situation de mon cœur, qu'effectivement je me sentis prête à m'évanouir, en disant presto non son piu forte. Certes la musique avait ici changé d'expression : au lieu de la plainte coquette de Zerline, c'était le cri de la détresse la plus profonde; en ce moment je sentis que le comte s'était rapproché de mon côté, sa main toucha ma main pendante près de moi, un voile de flamme s'abaissa sur mes yeux, je saisis la chaise de la comtesse M... et je m'y cramponnai [...]; mais lorsque nous reprîmes ensemble : andiam, andiam mio bene, je sentis son haleine passer dans mes cheveux, son souffle courir sur mes épaules; un frisson me passa par les veines, je jetai en prononçant le mot amor un cri dans lequel s'épuisèrent toutes mes forces, et je m'évanouis ${ }^{7}[\ldots]$

Plusieurs scènes similaires interviennent dans d'autres romans. Dans Amaury (I843), Madeleine, interdite de danse à cause de sa santé fragile, supplie son père de la laisser valser une fois avec son fiancé; cette danse (sans doute sur L'Invitation à la valse de $\mathrm{Weber}^{8}$ ) fait perdre connaissance à la jeune fille et déclenche une longue maladie'. Dans Le Père la Ruine (I860), une jeune campagnarde, Huberte, grisée par un bal champêtre, tombe au pouvoir d'un séducteur, le Parisien Richard ${ }^{\mathrm{IO}}$ (xvII, p. I78-I79). Dans La Fille du marquis, enfin (écrit en I869 et publié de façon posthume en 1872), le thermidorien

5. Essai sur la musique, I825. Cité par Fr. Claudon dans La Musique des romantiques, Presses universitaires de France, coll. «Écriture», 1992, p. 7.

6. Fr. Claudon, La Musique des romantiques, op. cit., p. 6.

7. Pauline, éd. A.-M. Callet-Bianco, Gallimard, coll. "Folio classique», 2002, chap. viII, p. II3. Les références suivantes, données entre parenthèses, renvoient à la même édition.

8. Jouée par Liszt devant Pauline, cette même valse amène chez elle un trouble extrême (Pauline, chap. viII).

9. Amaury [1907], Paris, A. Le Vasseur et Cie, chap. XviI, p. 28. Les références suivantes renvoient à la même édition.

Io. Le Père la Ruine, Cœuvres-et-Valsery, Ressouvenances, 2000, chap. xviI, p. I78-I79. Les références suivantes renvoient à la même édition. 
Barras triomphe d'Éva après qu'elle s'est mise au piano, comme le suggère sans ambiguïté la fin de cet épisode, rapporté par Éva dans son journal :

La première vibration des touches, si vague qu'elle fût, fit courir dans toutes mes veines un frisson fiévreux. [...]

On m’a reparlé tant de fois de cette improvisation qu'il fallait bien qu'elle eût quelque chose d'extraordinaire.

Comme toujours, elle me laissa mourante. [...]

J'entendis les voitures qui quittaient l'hôtel, puis la voix de ma femme de chambre qui me demandait si je voulais me mettre au lit.

Je m’appuyai à son bras, haletante, la tête renversée, et je gagnai ma chambre. [...]

Tout à coup la fenêtre s'ouvrit, je reconnus Barras.

J'étendis la main vers la sonnette, je voulus pousser un cri mais ma main fut arrêtée par une autre main, mon cri fut étouffé sous la pression de deux lèvres brûlantes.

Je retombai inerte et éperdue sur mon lit"

On assiste donc, tout au long de la production romanesque de Dumas, au retour d'un schéma où la musique suscite un ravissement finalement destructeur, alors qu'elle présente un caractère sentimental, voire érotique, et qu'elle engage le corps de la jeune fille, par le chant, la danse ou l'interprétation. C'est cette configuration récurrente, son mécanisme et ses possibles interprétations qui seront l'objet de notre questionnement, visant à comprendre la liaison intime de la musique avec le danger le plus grand, chez un auteur pourtant mélomane et au sein d'une époque où la musique est fortement valorisée.

\section{Le sort est jeté}

Dans tous les cas répertoriés, la musique est à l'origine de la catastrophe du roman. Dans Pauline, il s'agit de l'engagement de l'héroïne auprès d'Horace, qui s'avère un brigand sans foi ni loi et un assassin; quand Pauline, devenue sa femme, aura découvert ce secret, Horace - dans la pure tradition du roman gothique! - la séquestrera au fond d'une abbaye en ruine et tentera de l'empoisonner. Dans Amaury, la musique et la danse déclenchent une maladie fatale, qui tue la jeune Madeleine et pousse au désespoir son père et son fiancé. Dans Le Père la Ruine, le bal champêtre cause le déshonneur d'Huberte, qui se suicide en entraînant dans la mort un jeune artiste, Valentin, sincèrement amoureux d'elle. Dans La Fille du marquis, l'improvisation à

II. La Fille du marquis [1907], Paris, A. Le Vasseur et Cie, chap. IX, p. 380. Les références suivantes renvoient à la même édition. 
laquelle Éva se livre au piano l'amène à succomber à Barras; or cette infidélité envers Jacques Mérey, son fiancé et son sauveur, équivaut à un véritable péché originel, qui ne sera pardonné qu'à la fin du roman, au terme d'un long processus de rédemption. Parmi les œuvres de Dumas où la musique déclenche la catastrophe du roman, le cas de Joseph Balsamo (I846-I848) aurait enfin pu être mentionné : la jeune Andrée de Taverney y est magnétisée par Cagliostro alors qu'elle improvise au clavecin; par la suite, c'est au cours d'un sommeil magnétique qu'elle est violée par Gilbert, un roturier qu'elle méprise. Elle tombe enceinte à la suite de ce viol, ce qui entraîne une grande part des péripéties du roman et, au-delà, du cycle les Mémoires d'un médecin dont il fait partie (cycle comprenant Joseph Balsamo, mais aussi Le Collier de la reine, Ange Pitou et La Comtesse de Charny).

La musique sert ainsi chez Dumas d'accélérateur ou de catalyseur narratif; elle court-circuite le récit en livrant le personnage à un ennemi, qui, autrement, n'aurait eu raison de lui que par de longs stratagèmes, ou pas du tout. Elle joue donc le même rôle que le merveilleux dans les contes ${ }^{12}$, et de fait, c'est à une forme de magie qu'elle est assimilée. La métaphore de l'envoûtement et l'image de la sirène sont à l'arrière-plan d'un épisode du Père la Ruine dans lequel le protagoniste, François Guichard, est séduit par le chant d'une lavandière : «il fût volontiers resté toute la nuit à écouter celle dont les accents l'avaient charmé» (I, p. I6). De même, dans le roman auquel il donne son nom, Amaury évoque "l'influence magique» d'une mélodie de Weber sur Madeleine (xxIx, p. 40), et Pauline souligne «l'effet magique» produit sur elle par une valse du même compositeur (Pauline, viII, p. IIO).

Mais la musique, qui prive les personnages de leur libre arbitre pour les jeter au pouvoir de forces occultes, n'a rien d'une magie blanche; c'est bien plutôt un ensorcellement qui aboutit à la folie du personnage, qu' elle arrache à lui-même et fait agir au rebours de sa volonté. C'est ce que souligne ce passage du Père la Ruine:

Le bal complétait la fascination. Nous connaissons déjà par Huberte le trouble et l'émotion qu'il portait dans son âme; encore la fade contredanse qu'elle avait essayée en plein jour [...] n'avait-elle rien du charme et de la puissance de cette fête; ces demi-ténèbres, ces bruits d'orchestre, ce concert de chants et de rires $[\ldots]$, tout contribuait à porter jusqu'au désordre le trouble de son cœur. Ce désordre était tel que, par moments, sous l'empire d'une effrayante

I2. Voir T. Todorov, Introduction à la littérature fantastique, Seuil, coll. «Points», I970, p. I7I et 174 : «Pour que la transgression de la loi provoque une modification rapide, il est commode que des forces surnaturelles interviennent"; "L'élément merveilleux se trouve être le matériel narratif qui remplit au mieux cette fonction précise : apporter une modification à la situation précédente, et rompre l'équilibre (ou le déséquilibre) établi.» 
surexcitation nerveuse, sa joie dégénérait en souffrance; il lui semblait que sa tête allait se fendre, que sa cervelle allait en jaillir, et cependant il lui était impossible de trouver en elle la force de s'arracher à ces funestes émotions. Elle valsait; elle était pâle, ses yeux se voilaient par instants, puis se rouvraient en lançant des éclairs dans les tourbillons de la valse; une partie de sa belle chevelure s'était dénouée et flottait autour de sa tête comme une auréole transparente. (XVII, p. 178-179.)

Cette chevelure flottante symbolise la perte de toute retenue chez Huberte, et elle évoque aussi l'image d'Ophélie, héroïne frappée par la folie dont Huberte, en se noyant, va connaître la tragédie. L'isotopie de la folie domine également cet extrait d'Amaury, où le héros se laisse entraîner par Madeleine dans une valse frénétique. Tout en opérant la transfiguration des personnages, et en les jetant dans un monde de délices célestes ${ }^{13}$, la musique les aliène totalement :

Alors l'ivresse qui s'était emparée de Madeleine le gagna à son tour : la recommandation que lui avait faite M. d'Avrigny [de ménager Madeleine], la promesse par laquelle il lui avait répondu, tout cela s'échappa de sa mémoire pour faire place à un délire étrange, inouï, inconnu; tous deux semblaient voler sur cette mesure fiévreuse, et cependant, à chaque instant, Madeleine murmurait : «Plus vite, Amaury, plus vite!» et Amaury obéissait; car ce n'était plus la pâle et languissante Madeleine qui lui parlait ainsi, c'était une jeune fille éclatante et radieuse, dont les yeux jetaient des flammes, dont le front était couronné de toutes les lueurs de la vie. [...] ils allaient toujours plus vite, ne voyant plus rien, n'entendant plus rien $[\ldots]$.

Tous deux paraissaient ne plus appartenir à la terre, emportés qu'ils étaient par un rêve divin, dans un tourbillon d'amour et de bonheur; tous deux s'inondaient de leurs regards, tous deux d'une voie haletante disaient : Je t'aime, je t'aime! et tous deux, puisant dans ce seul mot des forces nouvelles et presque insensées, précipitaient encore le mouvement, espérant qu'ils allaient mourir ainsi, ne se sentant plus de ce monde, se croyant au ciel.

Tout à coup Madeleine pesa de tout son poids au bras d'Amaury, il s'arrêta. Pâle, ployée, renversée en arrière, les yeux fermés, la lèvre entrouverte, elle était évanouie. (xvII, p. 28.)

La musique est donc un charme le plus souvent maléfique.

13. Dans ce passage, la musique apparaît aussi, conformément à la vision romantique, comme le langage de l'amour par excellence, permettant à ceux qui l'écoutent de communier dans une même pensée. Voir A. de Lamartine, Cours familier de littérature: "Il y a dans la musique une langue sans parole, qui permet [...] de tout dire sans rien exprimer" (Cité par R. L. Evans dans Les Romantiques français et la musique, op. cit., p. 90. 


\section{L’âme et le corps, séparés}

Le modèle dominant qui s'impose néanmoins à Dumas pour figurer la musique n'est pas celui de la magie traditionnelle, mais celui du magnétisme ${ }^{14}$ (qui suscite un engouement encore très fort pendant toute la première moitié du XIX ${ }^{e}$ siècle). Ces deux forces se conjuguent pour déposséder d'elle-même Andrée de Taverney, dont la première magnétisation s'accomplit alors qu'elle est joue du clavecin, comme si la musique favorisait le basculement dans un monde surnaturel :

L'air que jouait la jeune fille était mélancolique. Au reste, c'étaient plutôt de simples accords qu'un air. Elle improvisait sans doute, et repassait sur le clavecin les souvenirs de sa pensée ou les rêves de son imagination. [...]

Tout à coup, $[\ldots][\mathrm{t}]$ oute sa personne frissonna comme secouée d'une commotion électrique. [...]

Elle voulut se retourner, crier.

Mais Balsamo étendit ses bras en avant, et elle ne bougea plus. [...]

Andrée tenta encore une fois de se lever, mais elle ne put y parvenir : une force invisible, un engourdissement qui n'était point sans charme la clouèrent sur son fauteuil $[\ldots]$.

Elle fit pour appeler au secours un effort surhumain : sa bouche s'ouvrit, mais Balsamo étendit ses deux mains au-dessus de la tête de la jeune fille, et aucun son ne sortit de sa bouche. [...]La jeune fille n'avait plus ni force ni volonté; elle laissa retomber sa tête sur son épaule

Plus qu'une relation métaphorique, il y a comme une identité de nature entre le magnétisme et la musique, qui semble être pour Dumas une forme de magnétisme : elle plonge le sujet dans le même état, produit sur lui les mêmes effets. Dans Les Mohicans de Paris, le narrateur dit ainsi des voix de contralto qu'elles «s'emparent [... de de l'âme des spectateurs avec la rapidité et la force de l'électricité ou du magnétisme ${ }^{16}{ }^{\prime}$. Dans La Fille du marquis, Éva décrit en ces termes les effets de la musique :

La musique avait sur moi cette influence fatale qu'elle m'enlevait le reste de ma raison. Quand je tombais dans cet état cataleptique qui était presque toujours la suite de mes improvisations, j'étais littéralement à la merci de ceux avec qui je me

I4. La doctrine du magnétisme animal, supposant l'existence d'une propriété du corps animal le rendant réceptif à l'influence des corps célestes et à celle des corps environnants, a été élaborée au XVIII ${ }^{\mathrm{e}}$ siècle par le médecin allemand Franz Mesmer (I734-18I5). Fondateur d'une thérapeutique magnétique, il s'était établi à Paris dès février 1778 (au moment où les Parisiens se passionnaient pour la bouteille de Leyde et autres expériences relatives à l'électricité) et y avait obtenu un grand succès.

I5. Joseph Balsamo, éd. C. Schopp, Robert Laffont, coll. «Bouquins», I990, chap. VII, p. IIIII2.

16. Les Mohicans de Paris [1854-1855], éd. C. Schopp, Gallimard, coll. "Quarto», I998, chap. CLXXII, p. I380. 
trouvais. [...] On sait, du moment que j'étais mise en contact avec cet instrument [le piano], dans quel état d'exaltation magnétique j'entrais. (Ix, p. 355-378.)

Or, comme le magnétisme, la musique est foncièrement ambivalente. De même aussi que la passion, selon la conception romantique, est une énergie à la fois source de vie et source de mort (puisqu'elle accélère la vie tout en l'intensifiant), la musique peut tuer une personne faible, qui s'y adonne avec outrance, mais elle peut aussi être employée, à bon escient comme thérapie. Alors qu'il l'a recueillie encore petite, et presque débile, Jacques Mérey parvient ainsi à sortir Éva de son apathie en lui jouant à l'orgue un air de Cimarosa : «il put voir [...] Éva tressaillir [...], venir à lui comme le magnétisé vient au magnétiseur ${ }^{17}$ ». Peu après, Éva, «à la suite d'une commotion électrique qui l'avait enveloppée d'un fluide vivifiant ${ }^{18}$ ", se met d'elle-même au piano, et, à l'«éternel étonnement» de Jacques, joue un air entendu la veille. Lors de la maladie de Madeleine dont nous avons parlé, Amaury parvient également à lui redonner quelques forces en lui jouant la même valse de Weber, qui cette fois opère un effet bénéfique. Il raconte :

Il semblait que cette poétique organisation s'animât à l'harmonie, et reprît ses forces, à mesure que le mouvement s'accentuait.

Après s'être tenue un instant debout, [...] je la vis se mouvoir, et cette frêle malade, qu'à grand'peine son père et sa gouvernante avaient conduite de son lit à son fauteuil, s'avança lentement, mais d'un pas sûr, marchant sans bruit comme une ombre, mais sans chercher d'appui ni aux meubles, ni à la muraille. (Amaury, xxi, p. 33.)

Madeleine, à qui son père va répondre avec sévérité qu' "il ne faut pas abuser de cette puissance», s'écrie alors : «Ah! [...] c'est une magie. Mais voilà l'effet de la musique sur moi : je crois que si j'étais morte, il y a certains airs qui auraient la puissance de me tirer du tombeau.»

Comme le magnétisme thérapeutique pratiqué par Mesmer, la musique peut soigner en favorisant la circulation du fluide vital qui emplit les corps, et dont l'obstruction est cause de toutes les maladies selon le médecin allemand. Dans Le Collier de la reine, Dumas montre d'ailleurs ce dernier accompagner de musique ses cures collectives:

Alors [après que l'ambiance avait été réchauffée et imprégnée de parfums] on voyait les malades s'abandonner à l'impression toute voluptueuse de cette atmosphère, lorsque soudain une musique suave et vibrante, exécutée par des instruments et des musiciens invisibles, se perdait comme une douce flamme au milieu de ces parfums et de cette chaleur.

[...] cette musique frappait les nerfs avec une puissance irrésistible. On eût dit un de ces bruits mystérieux et inconnus de la nature qui étonnent et

17. Le Docteur mystérieux [1872], Slatkine, coll. «Ressources», I980, chap. viII, p. 86.

I8. Ibid., XXXIII, p. 8I. 
charment les animaux eux-mêmes, une plainte du vent dans les spirales sonores des rochers. [...]

Sur tous les visages que la surprise avait animés d'abord, se peignait peu à peu la satisfaction matérielle, caressée par tous ses endroits sensibles. L'âme cédait; elle sortait de ce refuge où elle se cache quand les maux du corps l'assiègent, et se répandant libre et joyeuse dans toute l'organisation, elle domptait la matière et [la] transformait ${ }^{19}$.

On retrouve ici un écho de l'idée, remontant à l'Antiquité, d'une musique source d'harmonie universelle, régissant le macrocosme et le microcosme. Cette idée se retrouve, christianisée, chez plusieurs romantiques comme Lamartine, qui écrit dans son Cours familier de littérature: "Dieu n'est pas seulement le grand architecte, le grand mathématicien, le grand poète des mondes, il est aussi le grand musicien. La création est un chant dont il a mesuré la cadence et dont il écoute la mélodie ${ }^{20}$.»

Cependant, chez Dumas, la musique favorise moins les échanges entre l'âme et le corps, comme dans l'extrait du Collier de la reine que je viens de citer, qu'elle n'opère au contraire leur séparation - à la manière du magnétisme, toujours. Libérée de son linceul, il est vrai que l'âme nage dans une extase céleste, et assouvit son aspiration vers l'infini en découvrant par avance le monde supraterrestre ${ }^{21}$. Dans un autre roman, Le Maître d'armes, alors que le héros écoute l' "harmonie merveilleuse» produite par un concert de cors, sur la Néva, il commente ainsi : «je fus, je l'avoue, ravi comme en extase ${ }^{22}$ ». Et dans Les Mohicans de Paris, pour Carmélite, qui est entrée dans l'art lyrique comme en religion, la musique est une sphère idéale dans laquelle elle rejoint, grâce au "merveilleux miracle de sa voix ${ }^{23}$ ", son amant défunt, Colomban.

Mais cette sphère idéale est donc aussi le royaume des morts, et Carmélite, dont le nom est éloquent, apparaît comme une morte-vivante. Parce que la musique détache l'âme du corps, elle est une initiation à la mort ${ }^{24}$; elle peut mener à elle, et l'on voit un premier danger lié à la séparation entre l'âme et le corps se profiler ici.

19. Le Collier de la reine [1849-1850], éd. C. Schopp, Robert Laffont, coll. "Bouquins», I990, chap. XvII, p. 173.

20. Cité par R. L. Evans dans Les Romantiques français et la musique, op. cit., p. 30.

2I. Une pareille conception est répandue chez les romantiques : pour Vigny, la musique est ainsi «l'écho d'un autre monde» (Lettre au marquis de la Grange, 2 février I829. Cité par R. Leslie Evans dans Les Romantiques français et la musique, op. cit., p. I8). George Sand écrit de même à propos de la Symphonie pastorale qu'elle ouvre «des perspectives enchantées [...], des horizons sans limites." (Lettre d'un voyageur à Meyerbeer, septembre I836, cité par R. L. Evans, op. cit., p. 94).

22. Le Maître d'armes [I840-184I], Éditions des Syrtes, coll. «Joyaux oubliés», 2002, chap. II, p. 26.

23. Éd. citée, chap. CLXXII, p. I380.

24. "L'art est un plaisir austère et qui prépare merveilleusement la mort", lit-on dans Amaury (xxxII, p. 4I). 


\section{Musique et hystérie}

Mais la musique est surtout dangereuse parce qu'en arrachant l'âme du corps, elle laisse celui-ci sans gardien. L'individu peut alors se laisser entraîner par sa sensualité à des actes contraires à la raison. En des termes plus modernes (et bien sûr anachroniques), un refoulé sexuel déborde : Pauline interprétant Zerline se livre à Horace-Don Giovanni; Éva, improvisant au piano au gré de ses passions, alors qu'elle est habitée par le rôle de Juliette, cède au désir de Barras-Roméo, et sans doute à son propre désir; la vierge farouche, Andrée de Taverney, se laisse subjuguer par Joseph Balsamo, alors qu'elle improvise elle aussi au clavecin, suivant son caprice. Huberte, enfin, tout entière au plaisir de la danse, se donne à Richard malgré son amour platonique pour Valentin. Qualifiée par Dumas d' "art sensuel» dans "Poètes, peintres et musiciens", une causerie recueillie en I86I dans Bric-à-brac, la musique semble révéler à l'homme ses désirs inconscients, conformément au sentiment de Raymond Leslie Evans, selon qui la conception de la musique comme «langage de la vie subconsciente» est «l'apport le plus important des romantiques à la psychologie $\mathrm{e}^{25}$ ».

Cependant, ce déchaînement sensuel que fait naître la musique est loin d'être bénéfique, et les comportements véritablement pathologiques qui éclatent chez les personnages de Dumas nous indiquent que sa condamnation va au-delà d'un vulgaire moralisme littéraire. La censure, au sens psychanalytique, semble en réalité le sujet même des scènes de ravissement musical et la vraie cause du trouble extrême qui submerge les personnages. Ces jeunes filles en proie à l'extase musicale montrent en effet des symptômes très proches de ceux qui se font jour lors des crises d'hystérie, maladie nerveuse surtout féminine (et conçue comme exclusivement féminine jusqu'à Charcot) qui se caractérise par une somatisation de conflits psychiques, et se manifeste par des attaques, des convulsions, des évanouissements, des crises cataleptiques ou encore des états somnambuliques, autant de signes qu'on retrouve chez Pauline, Éva ou Andrée de Taverney.

Quel conflit, dans leurs cas, ces états traduisent-ils? Celui entre un désir sexuel refoulé et un devoir moral, dont la musique, comme le magnétisme, symboliserait la contradiction en séparant l'âme du corps? Ces états ne manifestent-ils pas un traumatisme plus profond, un conflit plus violent? Sans soumettre les personnages de Dumas à une psychanalyse en règle, on signalera quelques faits troublants, qui tendent à révéler chez ces personnages, au-delà des symptômes, les causes profondes de l'hystérie. D'abord, toutes ces jeunes

25. Op. cit., p. IX. 
filles sont vierges avant d'être ravies; ensuite, certaines sont étonnamment androgynes, notamment Andrée de Taverney, dont le prénom signale la virilité latente. La frigidité de ce personnage, constamment comparé à une statue de marbre, est par ailleurs évidente. Toutes ces jeunes filles, en outre, entretiennent une relation trouble avec la figure paternelle. Soit cette figure est totalement absente, comme pour Pauline, soit c'est une figure incestueuse : il est ainsi dit de manière explicite, au sujet du père de Madeleine d'Avrigny, qu'il est amoureux de sa fille; le père d'Andrée de Taverney tente de prostituer sa fille à Louis XV; quant à Éva, dans La Fille du marquis, elle a été élevée par Jacques Mérey, avec qui, devenue adulte, elle s'est fiancée. On rappellera enfin la liaison intime des scènes de ravissement musical avec le motif du viol. Ce motif est présent de façon paradigmatique ou métaphorique dans toutes les scènes évoquées, mais il est aussi présent de façon métonymique ou syntagmatique, puisqu'un acte charnel non librement consenti suit toujours l'extase née de la musique (sauf dans le cas de Madeleine d'Avrigny). Tout cela renvoie de façon assez convaincante aux origines de l'hystérie, rattachée par les psychiatres au complexe d'Edipe et à un traumatisme vécu par l'enfant à l'âge dit phallique.

Cette configuration dumasienne permet donc de prendre du recul par rapport au lieu commun d'une musique célébrée de manière univoque par les romantiques. C'est bien sûr en raison du danger des passions que la musique, art pathétique par excellence, est jugée périlleuse. La musique conduit cellesci à leur exacerbation, comme le souligne Éva lorsqu'elle évoque ses improvisations au piano :

Je me sentais frissonner et frémir à ces mélodies inconnues qui s'éveillaient sous mes doigts; ce n'était plus des notes, c'étaient des pleurs, des soupirs, des sanglots, des retours à la joie, à la vie, au bonheur, un hymne de reconnaissance à Dieu; je ne vivais plus de ma vie ordinaire, mais d'une vie convulsive et fiévreuse où se résumait comme sensation tout ce que j'avais éprouvé, ressenti, souffert depuis un mois. [...] J'étais à moi seule le chœur et les personnages d'une tragédie antique. (La Fille du marquis, IX, p. 338.)

Or les passions tuent, comme le prouve l'allégorie balzacienne de La Peau de chagrin (I83I). C'est pourquoi la musique peut être fatale, comme l'illustre encore un conte d'Hoffmann, Le Violon de Crémone (I8I2), que Dumas cite à de nombreuses reprises et où l'on voit l'héroïne, Antonia, mourir d'avoir atteint la perfection du chant. De même, la musique brise l'organisation trop fragile de Madeleine d'Avrigny, après avoir ranimé ses forces un moment. On peut néanmoins formuler l'hypothèse qu'au-delà d'un discours sur le danger 
des passions (discours qui tend à se médicaliser au cours du $\mathrm{XIX}^{\mathrm{e}}$ siècle $^{26}$ ), on trouve chez Dumas une sorte de prescience romanesque d'un trouble psychiatrique que Charcot et Babinski, puis Freud et Breuer, analyseraient bientôt en détail. C'est en partant d'une hypothèse semblable que les médecins aliénistes, sous le Second Empire, se saisirent par exemple du personnage d'Emma Bovary pour décrire la folie hystérique ${ }^{27}$, ou que d'autres trouvèrent dans Une histoire sans nom de Barbey d'Aurevilly (I882) la peinture de ce que Jean Bernard appellerait, du nom de l'héroïne aurévillienne, le «syndrome de Lasthénie de Ferjol ${ }^{28} »$. De la même façon, Dumas semble figurer par les douloureuses extases musicales de ses personnages un état névrotique dû aux mécanismes inconscients. En définitive, la puissance de la musique est exaltée une nouvelle fois, puisqu'elle symbolise cette jouissance suprême, perçue comme un risque de désintégration et de folie, dont le sujet tente de se protéger par des manifestations somatiques sans lien organique évoquant le processus de conversion hystérique ${ }^{29}$.

26. Dès 1838, dans Des maladies mentales considérées sous les rapports médical, hygiénique et médico-légal, J.-É. Esquirol fait des passions l'origine de l'aliénation mentale.

27. Voir S. Lepastier, «Hystérie : traces corporelles, paroles affectées et textes écrits», communication au colloque "Médecine, sciences de la vie et littérature en France en en Europe, de la Révolution à nos jours", université Stendhal - Grenoble 3, I2-I5 mars 2008.

28. Voir la communication de P. Noir, «L'hystérique ou la médicalisation du péché dans l'œuvre de Barbey d'Aurevilly», au colloque "Médecine, sciences de la vie et littérature en France en en Europe, de la Révolution à nos jours", université Stendhal-Grenoble 3, I2-I5 mars 2008. Ce syndrome désigne un cas d'anémie due à des hémorragies provoquées, et appariées aux saignements menstruels.

29. Voir J.-D. Nasio, L'hystérie ou l'enfant magnifique de la psychanalyse, Petite Bibliothèque Payot, 200I, p. 29: «souffrir sur un mode hystérique, c'est souffrir consciemment dans le corps, c'est-à-dire convertir la jouissance inconsciente et intolérable en souffrance corporelle». 
